\title{
Lambl's Excrescences: Current Diagnosis and Management
}

\author{
Ganesh Kumar K. Ammannaya
}

\begin{abstract}
Lambl's excrescences (LEs) are unusual, yet significant etiology of thromboembolism. LEs are fibrous valvular strands typically occurring at coaptation lines of the left-sided valves. These occur from wear and tear of the valves and comprise of a dense core of collagenous and elastic fibrils enclosed by endothelium. Transesophageal echocardiography (TEE) remains the gold standard in its diagnosis. Asymptomatic LEs are closely monitored, while symptomatic lesions with history of thromboembolism are managed with antiplatelet drugs or are anticoagulated. Surgery is indicated in case of recurrent thromboembolic episodes occurring while on medications.
\end{abstract}

Keywords: Lambl's excrescences; Valvular strands; Thromboembolism; Transesophageal echocardiography

\section{Introduction}

Dr. Vilem Dusan Lambl first reported Lambl's excrescences (LEs) in 1856, as filiform valvular fronds with hypermobility [1]. These cardiac processes ( $\leq 2 \mathrm{~mm}$ thick and $\geq 3 \mathrm{~mm}$ long), classically occur at the valve closure lines. LEs occur most commonly on the atrial side of the mitral valve $(68-76 \%)$, followed by the aortic valve on the ventricular side (38-50\%). LEs have also been shown to occur on prosthetic valves, and the affection of the right-sided native valves is extremely rare $[2,3]$. These strands have equal incidence in men and in women.

LEs originate on the endocardial surface owing to trivial endothelial injury caused by valvular wear and tear [4-6]. Though mostly asymptomatic, LE have been shown to be associated with thromboembolic events such as cerebrovascular accidents (CVA), peripheral embolization and acute coronary syndrome. These are more common in the excrescences of the aortic valve [5].

Manuscript submitted May 28, 2019, accepted June 25, 2019

Department of Cardiovascular and Thoracic Surgery, Lokmanya Tilak Municipal Medical College and General Hospital, Sion, Mumbai 400022, India. Email: doc.ammannaya@gmail.com

doi: https://doi.org/10.14740/cr892

\section{Pathology}

High stress in closure lines of left-sided valves can affect a tear or a lesion in the endocardium that initiates the pathogenesis of LE. Deposition of fibrin over this injured endocardium at the surface of the valve causes endothelial overgrowth, resulting in the development of papillary outgrowths. These partially detach from the surface, hyalinize, and ultimately become fibrosed. The increasing shear forces with age further contribute to their occurrence. A cluster of LE may separate from the valve surface, leading to thromboembolism. These lesions can occur in association with rheumatic heart disease, past history of endocarditis and pulmonary or systemic hypertension, but may also occur in otherwise healthy individuals $[5,7-9]$.

LEs are thin ( $\leq 2 \mathrm{~mm}$ in width) and elongated $(5-10 \mathrm{~mm}$ in length). Some excrescences, known as giant LEs, can be up to $2.5 \mathrm{~cm}$ in length. Two well-defined types of LEs can occur depending on the affected age group: lamellar excrescences which are seen more commonly in patients younger than 30 years, while filiform excrescences which occur in older patients [10].

Histologically, LEs comprise of a connective tissue center containing copious collagen and elastic fibrils, enclosed by a single layer of endothelium. The densely hyalinized central core is lined by bland-looking, endocardial-type spindle cells. The arrangement of tissues is in apposed layers, with variable collagen fibril orientation [7-11].

\section{Diagnosis}

Most patients with LE are asymptomatic and the valvular strands are found incidentally on echocardiography [2]. However, they can be a rare cause of stroke, or coronary artery obstruction [12]. LE, particularly those found on aortic and mitral valve leaflets can break free and embolize to the brain causing stroke. The potential cause of a CVA should be identified by Doppler ultrasonography of the carotid arteries and by echocardiographic scanning of the ascending, transverse, and arch segments of the aorta [3, 4].

Transesophageal echocardiography (TEE) is recommended in causal evaluation of any stroke, because it is more sensitive than transthoracic echocardiography (TTE). However, with the development of the current high-resolution echo systems, valvular strands can often be detected 


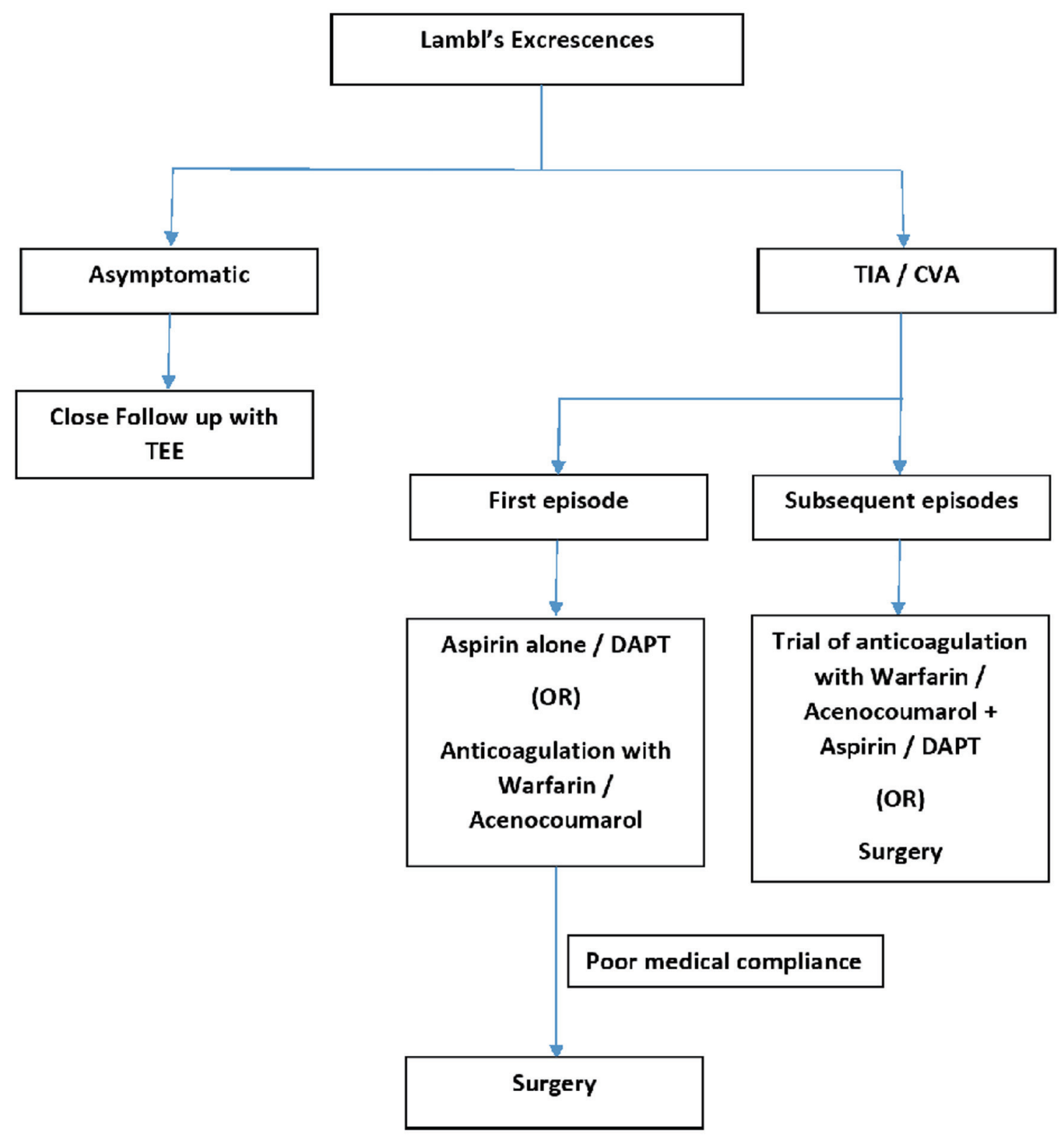

Figure 1. Current practices in the management of LE. TEE: transesophageal echocardiography; TIA: transient ischemic attack; CVA: cerebrovascular accident; DAPT: dual antiplatelet therapy.

on TTE [3]. On echocardiography, LE appears as independent, undulating hypermobile, strand-like structures $(\leq 2 \mathrm{~mm}$ thick and $\geq 3 \mathrm{~mm}$ long) at the leaflet's coaptation $[4,11,13$, 14].

The differential diagnosis for these excrescences includes imaging artifact, vegetations, thrombi, redundant leaflet, flap due to aortic dissection, papillary fibroelastoma, myxoma, other cardiac neoplasms and metastases. Of these, the most difficult is the distinction between LEs and fibroelastomas. Histopathologically, they are similar. However, fibroelastomas are larger, most often solitary, usually arise from the mid portion of valve leaflets and appear as pedunculated lesions on the less mechanically affected parts of valves, and on other areas of the endocardium. In contrast, LEs are multiple and arise from the line of valve closure $[4,15]$.

\section{Treatment}

Definitive guidelines for the management of patients with LE do not exist as of today, owing to rarity of the condition and a consequent paucity of well-designed studies. The various strategies employed in the management of LE include single and dual antiplatelet therapy, anticoagulation and surgery [16], and are largely based on isolated case reports.

Asymptomatic patients with LE should be closely moni- 
tored with TEE follow-up [2]. Patients who have LE and have experienced one episode of transient ischemic attack (TIA)/ CVA should be treated conservatively with antiplatelet therapy. While some authors report the use of aspirin alone [17, 18], others have advocated dual antiplatelet therapy (DAPT) with the addition of clopidogrel $[14,19]$. There is no general consensus in the literature about whether to use anticoagulation $[5,20]$ versus antiplatelet therapy in a patient with a single embolic episode with LE.

It is recommended to include LE in the differential diagnosis of cryptogenic stroke [14] and TEE is strongly advisable in this setting $[2,14]$. If LE is present, treatment with DAPT should be considered. If there is a recurrent ischemic event while on this therapy, a trial of anticoagulation therapy with warfarin or acenocoumarol should be considered, along with aspirin or DAPT before proceeding with surgical resection of the LE [14]. It is reasonable to offer surgical valve replacement after second CVA related to LE as the recurrence rate of CVA is high [15]. In a patient with poor medical compliance, surgical excision and valve replacement may be a better option for TIA/CVA symptoms secondary to LE, given the bleeding risk on antiplatelet/anticoagulation therapy [21]. A summary of the current practices in the management of LE is schematically represented in Figure 1.

There is a general consensus that the choice between surgical intervention versus conservative management, needs to be decided on a case-by-case basis and should be based on the pre-operative risk stratification for the patient [17].

\section{Conclusions}

LEs are unusual, but a proven differential diagnosis of cryptogenic stroke. TEE is still the gold standard in their diagnosis [14]. Our current understanding about the management of LE is drawn entirely from previous case reports. High-powered studies directly comparing the various modalities of treatment in different clinical settings are lacking. This emphasizes the need for statistically well-designed studies to further validate our current understanding about LE and its successful management. Till such a time, a case-specific approach with respect to decision making has to be adopted depending on the clinical status of the patient.

\section{Acknowledgments}

None.

\section{Financial Disclosure}

None to declare.

\section{Conflict of Interest}

None.

\section{Author Contributions}

GKKA: entire manuscript conceptualization, writing and editing.

\section{References}

1. Lambl VA. Papillare exkreszenzen an der semilunar-klappe der aorta. Wien Med Wochenscshr. 1856;6:244-447.

2. Aziz F, Baciewicz FA, Jr. Lambl's excrescences: review and recommendations. Tex Heart Inst J. 2007;34(3):366368.

3. Leitman M, Tyomkin V, Peleg E, Shmueli R, Krakover R, Vered Z. Clinical significance and prevalence of valvular strands during routine echo examinations. Eur Heart J Cardiovasc Imaging. 2014;15(11):1226-1230.

4. Jaffe W, Figueredo VM. An example of Lambl's excrescences by transesophageal echocardiogram: a commonly misinterpreted lesion. Echocardiography. 2007;24(10):1086-1089.

5. Kalavakunta JK, Peddi P, Bantu V, Tokala H, Kodenchery M. Lambl's excrescences: a rare cause of stroke. J Heart Valve Dis. 2010;19(5):669-670.

6. Al-Ansari S, Hindori V, Riezebos RK, Yilmaz A. Multiple Lambl's excrescences with subvalvular extension, a rare cause of cryptogenic stroke: treated by port-access cardiac surgery. BMJ Case Rep. 2013;2013.

7. Riddle JM, Wang CH, Magilligan DJ, Jr., Stein PD. Scanning electron microscopy of surgically excised human mitral valves in patients over 45 years of age. Am J Cardiol. 1989;63(7):471-477.

8. Nighoghossian N, Derex L, Loire R, Perinetti M, Honnorat J, Riche G, Barthelet M, et al. Giant lambl excrescences. An unusual source of cerebral embolism. Arch Neurol. 1997;54(1):41-44.

9. Aggarwal A, Leavitt BJ. Giant lambl's excrescences. N Engl J Med. 2003;349:25.

10. Hurle JM, Garcia-Martinez V, Sanchez-Quintana D. Morphologic characteristics and structure of surface excrescences (Lambl's excrescences) in the normal aortic valve. Am J Cardiol. 1986;58(13):1223-1227.

11. Roldan CA, Shively BK, Crawford MH. Valve excrescences: prevalence, evolution and risk for cardioembolism. J Am Coll Cardiol. 1997;30(5):1308-1314.

12. Dangas G, Dailey-Sterling FG, Sharma SK, Chockalingham S, Albanese JR, Reich DL, Meller J, et al. Non-Qwave infarction and ostial left coronary obstruction due to giant Lambl's excrescences of the aortic valve. Circulation. 1999;99(14):1919-1921.

13. Freedberg RS, Goodkin GM, Perez JL, Tunick PA, Kronzon I. Valve strands are strongly associated with systemic embolization: a transesophageal echocardiographic study. J Am Coll Cardiol. 1995;26(7):1709-1712.

14. Chu A, Aung TT, Sahalon H, Choksi V, Feiz H. Lambl's Excrescence Associated with Cryptogenic Stroke: A Case Report and Literature Review. Am J Case Rep. 2015;16:876-881. 
15. Daveron E, Jain N, Kelley GP, Luer WH, Fermin C, Helmcke F, Kerut EK. Papillary fibroelastoma and Lambl's excrescences: echocardiographic diagnosis and differential diagnosis. Echocardiography. 2005;22(3):285-287.

16. Kariyanna PT, Jayarangaiah A, Rednam C, Hegde S, Marmur JD, Kamran H, Wengrofsky P, et al. Lambl's excrescences and stroke: a scoping study. Int J Clin Res Trials. 2018;3(2):127.

17. Davogustto G, Fernando RR, Loghin C. Lambl's excrescence, migrainous headaches, and "tiger stripes": puzzling findings in one patient. Tex Heart Inst J. 2015;42(1):7072.
18. Wolf RC, Spiess J, Huber R. [Lambl's excrescence and cerebral ischemic insult]. Nervenarzt. 2006;77(12):14921494.

19. Ker J. The serpentine mitral valve and cerebral embolism. Cardiovasc Ultrasound. 2011;9:7.

20. Kamran H, Patel N, Singh G, Pasricha V, Salifu M, McFarlane SI. Lambl's excrescences: A case report and review of the literature. Clin Case Rep Rev. 2016;2(7):486488.

21. Osorio B, Hou L, Xu J, Pagan E, et al. Lambl's excrescences and a review of therapeutic strategies. Case Reports in Internal Medicine. 2018;5(3). 